\title{
Comparison of understorey bird species in relation to edge-interior gradient in an isolated tropical rainforest of Malaysia
}

\begin{abstract}
Forest fragmentation results in a loss of forest interior and an increase in edge habitat. As a result, these changes may affect the bird species distribution and composition. We studied how understorey bird community composition and habitat variables changed along an edgeto-interior gradient in a fragmented lowland rainforest in Peninsular Malaysia. Birds and environmental variables were recorded at each of the 93 sampling points distributed along the 14 parallel transects that were systematically placed across the gradient of distance from the forest edge to the forest interior. Species composition was different along the edge-interior gradient, although only a few species were strictly confined to either edge or interior habitat. Based on bird-habitat associations along the edge-interior gradient, some of these edgepreferred species occurred in high numbers at the matrix surrounding the patch. In contrast, the interior-specialist group, mainly terrestrial insectivores, avoided the forest edge and was positively associated with humidity, canopy cover, the number of dead trees, percentage of litter cover, and depth of the litter layer. Species endemic to the Sunda subregion were more abundant in the interior of the forest. From a conservation perspective, forest remnants in the lowlands of Peninsular Malaysia that have a deep leaf litter layer, dense canopy cover, high number of dead trees, and high relative humidity are able to support understorey bird species that are sensitive to edge effects. The forest has important conservation value even though it is fragmented and isolated.
\end{abstract}

Keyword: Understorey birds; Isolated forest; Diversity indices; Edge forest; Interior forest 